\title{
Oxygen content and inhomogeneity effects on the electrical properties of $\mathrm{YBa}_{2} \mathrm{Cu}_{3} \mathrm{O}_{y}$ thin films
}

\author{
R. Aguiar, F. Sánchez, and M. Varela \\ Departament de Física Aplicada i Electrònica, Universitat de Barcelona, Av. Diagonal 647, E-08028 \\ Barcelona, Spain
}

(Received 2 January 1995; accepted 16 August 1996)

Oxygen content is a very important factor influencing the electrical properties of $\mathrm{YBa}_{2} \mathrm{Cu}_{3} \mathrm{O}_{y}$. In this work the electrical properties of laser deposited $\mathrm{YBa}_{2} \mathrm{Cu}_{3} \mathrm{O}_{y}$ thin films on $\mathrm{LaAlO}_{3}(100)$, in the whole range $6 \leqslant y \leqslant 7$, are studied. An electrical network model, which randomly assigns oxygen contents and $R(T)$ characteristics to the different elements in the circuit according to an arbitrary distribution, is used to analyze several features in the measured $R(T)$ characteristics as a function of oxygen homogeneity. The model takes into account both short-range and long-range oxygen inhomogeneities. Good agreement between estimated oxygen contents from x-ray diffraction data in our samples and the average oxygen contents used to reproduce their $R(T)$ characteristics is found. The model points out that oxygen homogeneity is very important in order to get the best and reproducible properties, and for conduction and superconductivity analysis through the shape or derivatives of $R(T)$ characteristics.

\section{INTRODUCTION}

The strong dependence of the properties of $\mathrm{YBa}_{2} \mathrm{Cu}_{3} \mathrm{O}_{y}$ on the carrier concentration has been widely evidenced. This concentration can be controlled by doping the compound with oxygen atoms. $\mathrm{YBa}_{2} \mathrm{Cu}_{3} \mathrm{O}_{6}$ is a tetragonal semiconducting and nonsuperconducting material, but its properties can be modified by adding oxygen atoms to the $\mathrm{Cu}-\mathrm{O}$ chains. That is why the usual formula $\mathrm{YBa}_{2} \mathrm{Cu}_{3} \mathrm{O}_{y}(6 \leqslant y \leqslant 7)$ is used. The extra oxygen atoms introduce holes in the structure, which are responsible for the increase in the conductivity ${ }^{1}$ and the transition to a metallic behavior in the normal state. This behavior is not exactly linear and a roundup of the $R(T)$ characteristics is found in some cases. This fact is still under discussion by many authors and has sometimes been related to some specific transport properties in the high temperature superconducting cuprates. ${ }^{1}$

When the oxygen content of $\mathrm{YBa}_{2} \mathrm{Cu}_{3} \mathrm{O}_{y}$ is below 6.3-6.4 the material is still nonsuperconducting. When $6.4 \leqslant y \leqslant 6.7$ the crystal structure becomes orthorhombic (so-called orthorhombic II phase), and the material is already metallic in the normal state and superconducting with $T_{c}$ about $60 \mathrm{~K}$. If $y$ goes up, the critical temperature suddenly increases and remains about $90 \mathrm{~K}$ for the highest oxygen contents (orthorhombic I phase). Though this is the most common behavior, the two plateaus about $60 \mathrm{~K}$ and $90 \mathrm{~K}$ may disappear, depending on the sample preparation, due to the influence of oxygen ordering inside the crystal structure on the electrical behavior. ${ }^{2,3}$ Most previous work on the relationships between electrical properties and oxygen content in $\mathrm{YBa}_{2} \mathrm{Cu}_{3} \mathrm{O}_{y}$ has been carried out on bulk material ${ }^{3-5}$ and in fewer cases ${ }^{6,7}$ in thin films, deposited by different techniques on various substrates.

In this work, we study the electrical properties of $\mathrm{YBa}_{2} \mathrm{Cu}_{3} \mathrm{O}_{y}$ thin films on $\mathrm{LaAlO}_{3}$ (100), deposited under different conditions and in situ or ex situ annealing steps in order to obtain oxygen contents in the whole range between $y=6$ and $y=7$. An electrical network model which takes into account different kinds of oxygen inhomogeneities will be provided in order to explain some features of the $R(T)$ characteristics.

\section{EXPERIMENTAL}

$\mathrm{YBa}_{2} \mathrm{Cu}_{3} \mathrm{O}_{y}$ thin films were deposited on $\mathrm{LaAlO}_{3}(100)$ single crystals by laser ablation. The process was carried out with an ArF excimer laser ( $\lambda=193 \mathrm{~nm}, \tau=23 \mathrm{~ns}$ ), focused up to $3 \mathrm{~J} / \mathrm{cm}^{2}$ on a $\mathrm{YBa}_{2} \mathrm{Cu}_{3} \mathrm{O}_{7}$ target. The deposition temperature was changed between $600{ }^{\circ} \mathrm{C}$ and $750{ }^{\circ} \mathrm{C}$. Four kinds of samples were studied. The first group was deposited under 0.2 mbar of $\mathrm{O}_{2}$, and simply cooled down after deposition, under the same pressure, and the second one was cooled down in the same way in $1 \mathrm{~atm} \mathrm{O}_{2}$. A third series was deposited at 0.1 mbar $\mathrm{O}_{2}$, cooled in $1 \mathrm{~atm}$ $\mathrm{O}_{2}$ down to $450{ }^{\circ} \mathrm{C}$, and kept at this temperature for $15 \mathrm{~min}$. The last group is made up with some of those samples ex situ annealed in flowing oxygen at $450{ }^{\circ} \mathrm{C}$.

The samples were studied by $\mathrm{x}$-ray diffractometry (XRD), scanning electron microscopy (SEM), and electrical measurements. The electrical characterization was focused on room temperature conductivity and $R(T)$ characteristics. 


\section{STRUCTURAL PROPERTIES}

The films are 100 to $150 \mathrm{~nm}$ thick, with a dense, quite smooth surface, depending on the deposition temperature, and few droplets on them. The XRD analysis revealed that the films have the $c$ axis perpendicular to the surface whatever the deposition conditions. XRD pole figures show that the $\mathrm{YBa}_{2} \mathrm{Cu}_{3} \mathrm{O}_{y}$ films grow epitaxially with $a$ or $b$ axes parallel to the [100] direction of the substrate. Quantitative Raman spectroscopy analysis according to Thomsen et al. ${ }^{8}$ revealed that about $90 \%$ of the material had this orientation. Only a few small domains with $a / b$ axes rotated $45^{\circ}$ away from the [100] direction of the substrate were also found by $\mathrm{x}$-ray diffraction analysis in some samples deposited at the lowest temperatures. The crystalline quality of the films is shown by narrow rocking curves $\left(<1^{\circ}\right)$, but it is limited by the twin structure in the substrates which is sometimes reproduced in the film.

The $c$ parameter has been determined from the $(00 h)$ reflections in $\theta / 2 \theta$ analysis. The peaks were fitted with pseudo-Voigt functions, and the $c$ value was extrapolated by using the Nelson-Riley function. The oxygen content has been estimated from the $c$ parameter according to an average of several measurements given by different groups mainly for bulk material. ${ }^{5,9-13}$ The estimated oxygen contents ranged from 6.0 to $6.9( \pm 0.05)$ (Table I).

\section{ELECTRICAL CHARACTERIZATION}

\section{A. Room temperature conductivity}

The room temperature conductivity $(\sigma)$ was measured by the van der Pauw method. Due to the twinned

TABLE I. Oxygen content, room temperature conductivity, critical temperature, and transition width for $\mathrm{YBa}_{2} \mathrm{Cu}_{3} \mathrm{O}_{y}$ thin films on $\mathrm{LaAlO}_{3}(100)$.

\begin{tabular}{ccccc}
\hline \hline$y$ & $T_{c}(\mathrm{~K})$ & $T_{c 0}(\mathrm{~K})$ & $\Delta T_{c}$ & $\sigma\left(\mathrm{S} \cdot \mathrm{cm}^{-1}\right)$ \\
\hline 6.15 & $*$ & $*$ & $*$ & $2.0 \times 10^{1}$ \\
6.03 & $*$ & $*$ & $*$ & $2.0 \times 10^{1}$ \\
6.41 & 39.0 & 36.6 & 8.9 & $4.3 \times 10^{3}$ \\
6.25 & $*$ & $*$ & $*$ & $5.9 \times 10^{2}$ \\
6.49 & 42.6 & 41.2 & 4.9 & $1.5 \times 10^{3}$ \\
6.49 & 50.0 & 49.7 & 3.5 & $2.4 \times 10^{3}$ \\
6.59 & 54.0 & 53.8 & 1.6 & $2.8 \times 10^{3}$ \\
6.47 & 46.0 & 45.8 & 4.9 & $1.4 \times 10^{3}$ \\
6.52 & 46.0 & 41 & 11.0 & $6.5 \times 10^{2}$ \\
6.69 & 49.0 & 42.2 & 10.0 & $1.1 \times 10^{3}$ \\
6.83 & 55.0 & 22.0 & 26.8 & $5.1 \times 10^{3}$ \\
6.74 & 84.9 & 83.0 & 16.1 & $\cdots$ \\
6.80 & 85.2 & 80.3 & 5.3 & $\cdots$ \\
6.87 & 86.5 & 86.2 & 2.6 & $1.9 \times 10^{3}$ \\
6.72 & 77.3 & 68.5 & 9.4 & $1.5 \times 10^{3}$ \\
6.88 & 87.0 & 85.5 & 2.8 & $1.6 \times 10^{3}$ \\
\hline \hline
\end{tabular}

*Nonsuperconducting films. structure of the films, the obtained values mainly correspond to a combination of $\sigma_{a}$ and $\sigma_{b}$. The measured $\sigma$ values are between $2.0 \times 10^{1}$ and $4.3 \times 10^{3} \mathrm{~S} \cdot \mathrm{cm}^{-1}$, depending on the oxygen content of the films (Table I). The most significant change takes place when $y$ changes from 6.2 to 6.3 , when $\sigma$ suddenly increases almost by two orders of magnitude. For increasing oxygen contents the conductivity increases, but it also changes with the growth procedure, as far as growth process not only defines the oxygen content but also determines the grain size and film structure, and $\sigma$ depends on these properties, too.

\section{B. Oxygen content and $R(T)$ characteristics}

The resistance dependence on the temperature drastically changes with the oxygen content (Fig. 1). With $y \leqslant 6.3$ the samples are not superconducting. Their behavior is semiconducting or a combination of metallic behavior at high temperatures and semiconducting at low temperatures. The activation energy of the semiconducting curves is about $1-4 \mathrm{meV}$, and it usually decreases when the oxygen content increases.

When the oxygen content increases over 6.3, the films become approximately metallic, and superconducting. The temperature where the maximum slope at the transition is found (here named critical temperature $T_{c}$ ) is about $50 \mathrm{~K}$ if $y<6.7$ and increases up to $87 \mathrm{~K}$ when $y$ is over this value (Fig. 2). The zero resistance temperatures $\left(T_{c 0}\right)$ are, respectively, about $48 \mathrm{~K}$ and $86 \mathrm{~K}$.

The transition width $(90 \%-10 \%$ criterium) also changes with the oxygen content, leading to the doublepeaked curve in Fig. 3. The maximum widths are found when the oxygen content approaches the value where a phase transition occurs. This can be easily explained from the two-plateau dependence of $T_{c}$ on $y$ (Fig. 2). If there is a small inhomogeneity in the oxygen content, then different areas in the film would have different $T_{c}$. If the oxygen content is close to a phase transition (semiconducting to low $T_{c}$ superconductor, or low $T_{c}$ to high $T_{c}$ superconductor), then the critical temperatures of different sample areas will be very different, leading to wide transitions. But if $y$ is far away from these values, then the critical temperature is almost uniform and the transition width is much narrower.

Both the ratio $R(300 \mathrm{~K}) / R(100 \mathrm{~K})\left(\right.$ here $\left.R_{300} / R_{100}\right)$ and the transition width are commonly used as quality factors for $\mathrm{YBa}_{2} \mathrm{Cu}_{3} \mathrm{O}_{y}$ films. The slope in the normal state is mainly related to the relative amounts of conducting phases and semiconducting phases, and the contribution of defective areas which lead to residual resistivity. The higher slopes in the normal state usually correspond to the narrower transitions, as can be seen in Table I. 

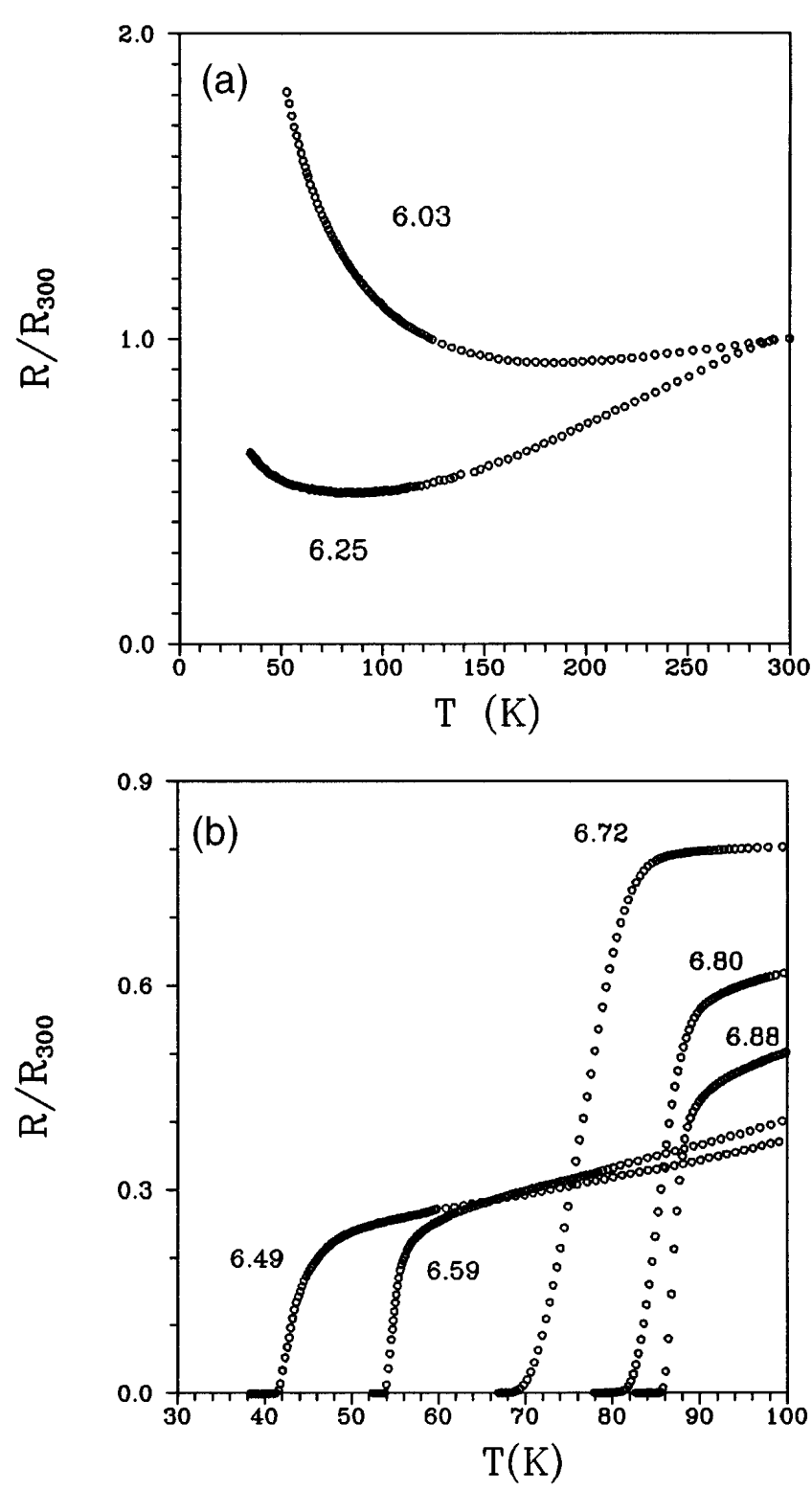

FIG. 1. Normalized resistance versus temperature for different oxygen contents, corresponding to films deposited by different steps. (a) Nonsuperconducting samples and (b) superconducting samples.

\section{MODELING THE $R(T)$ CHARACTERISTICS; DISCUSSION}

\section{A. Random resistor model}

The main $R(T)$ characteristics of $\mathrm{YBa}_{2} \mathrm{Cu}_{3} \mathrm{O}_{y}$ may be described by a few features as follows: (i) The normal state behavior can be either metallic or semiconducting, depending on the oxygen content. (ii) The critical temperature also depends on the oxygen content in the material. (iii) The transition to the superconducting state is not strictly abrupt and it is determined by several phenomena. When the temperature is still over $T_{c}$, the conductivity increases due to paraconductivity effects so that the $R(T)$ curve is then rounded down at $T>$

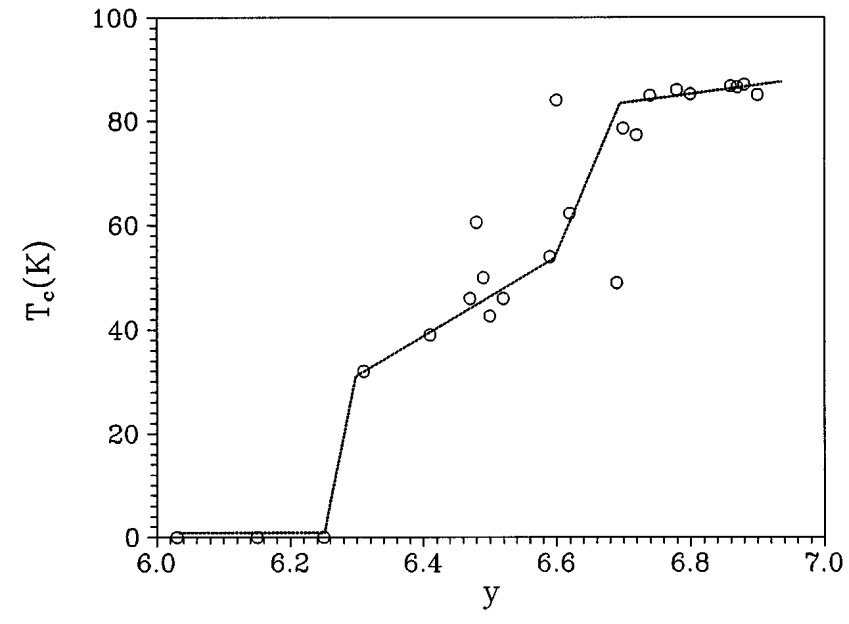

FIG. 2. Critical temperature versus oxygen content for films obtained by different processes.

$T_{c}{ }^{14-16}$ Below the superconducting onset temperature, the resistance in samples with oxygen inhomogeneities or grain boundaries decreases, due to the superconducting transition taking place in some areas. But entirely superconducting paths may not be formed until the temperature decreases further. Moreover, the critical current density across the grain boundaries may be too low to carry the measuring current in the superconducting state.

Some work has already been done by some groups in order to model the conducting behavior of semiconducting/metallic and superconducting material mixtures. In those works some pairs of resistors with well-defined $R(T)$ parameters were used in parallel to show the effect of mixing different phases in one sample. ${ }^{17-19}$

In order to study the influence of oxygen homogeneity in the conducting behavior, we have modeled the film as a random resistor network. Some resistances are grouped together in parallel, and then a series of these groups is built. In this case we used a series of

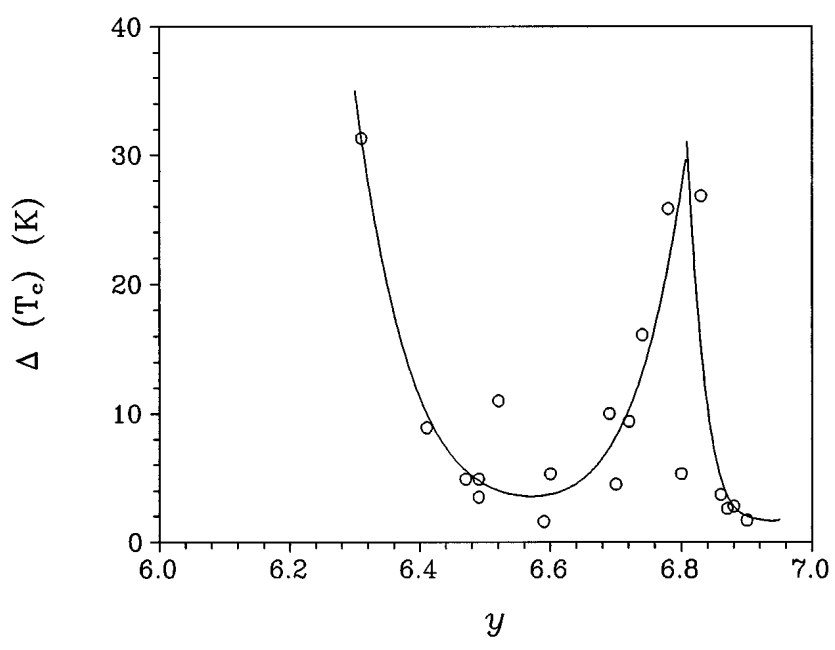

FIG. 3. Dependence of the transition width on the oxygen content. 
1000 groups of 10 parallel elements each, in order to improve statistics (see diagram in Fig. 4).

A random oxygen content value $y_{i}\left(6.0 \leqslant y_{i} \leqslant 7.0\right)$ is given to each resistor following a Gaussian probability distribution. As a result, an arbitrary Gaussian distribution of oxygen content values is obtained all over the whole resistor population, characterized by a mean $y$ value and a standard deviation. The mean $y$ and the standard deviation are input parameters at each run. In order to reproduce a long-range spatial variation in the average oxygen content, the probability distribution used for the oxygen content assignment can be gradually changed along the different resistor groups in series. In this case the average content and standard deviation of the oxygen statistical distribution are given at both end groups of the resistor network.

According to its oxygen content value, each resistor is given a specific $T_{c i}$ value where the transition starts, following the well-known $T_{c}-y$ curve obtained by Cava et al. for bulk material. ${ }^{22}$ Each oxygen content corresponds to $R_{i}(T)$ characteristics, which can be either purely metallic and superconducting or semiconducting as follows:

$y_{i}<6.40:$

$$
R_{i}(T)=\beta_{i} e^{\frac{E_{\rho}}{k_{B} T}}
$$

$y_{i} \geqslant 6.4:$

$$
\begin{aligned}
R_{i}(T)=R_{0}+\alpha_{i} T & \left(T \geqslant T_{c i}\right) \\
R_{i}(T)=\left(R_{0}+\alpha_{i} T_{c i}\right) e^{\gamma_{i}\left(T-T_{c i}\right)} & \\
\gamma_{i} \simeq \frac{3}{\Delta T_{i}} & \left(T<T_{c i}\right)
\end{aligned}
$$

The residual resistances $R_{0}$ and activation energy $E_{\rho}$ values are chosen at each run and set the same for all the elements. The $\alpha_{i}$ and $\beta_{i}$ parameters in the curves are computed so that all the individual resistances at $300 \mathrm{~K}$ would follow the empirical relationship for the conductivity:

$$
\sigma_{i}=\sigma_{0} e^{-\frac{7-y_{i}}{y_{0}}}
$$

where $y_{0}$ is about $0.2,{ }^{20,21}$ and $\sigma_{0}$ is the conductivity of $\mathrm{YBa}_{2} \mathrm{Cu}_{3} \mathrm{O}_{7}$.

The individual transition width $\left(\Delta T_{i}\right)$ is the same for all the resistive elements in the network that can become superconducting $\left(y_{i} \geqslant 6.40\right)$. Anyway, this value was always kept small $(0.1 \mathrm{~K})$, because these resistive elements are to be compared to small crystal domains or grains in a real sample, and the superconducting transition at these fractions could be expected to be as narrow as in a single crystal. That means that the parameters that are usually changed are $y$, its standard deviation, $R_{0}$ (which is set to zero in the following discussion), and $E_{\rho}$, which is expected to be of the order of some meV.

\section{B. Modeling the oxygen inhomogeneity}

This model allows study of the evolution of the $R(T)$ characteristics with the average oxygen content. Some results can be found in Fig. 5. As the average oxygen content increases, the $R(T)$ characteristics gradually change from semiconducting to metallic at high temperatures and semiconducting at low temperatures. When $y$ further increases, the $R(T)$ characteristics are metallic and superconducting with increasing critical temperature.

A small amount of superconducting elements mixed up with poor conductors can be enough to make the whole chain superconducting. That shows that a small
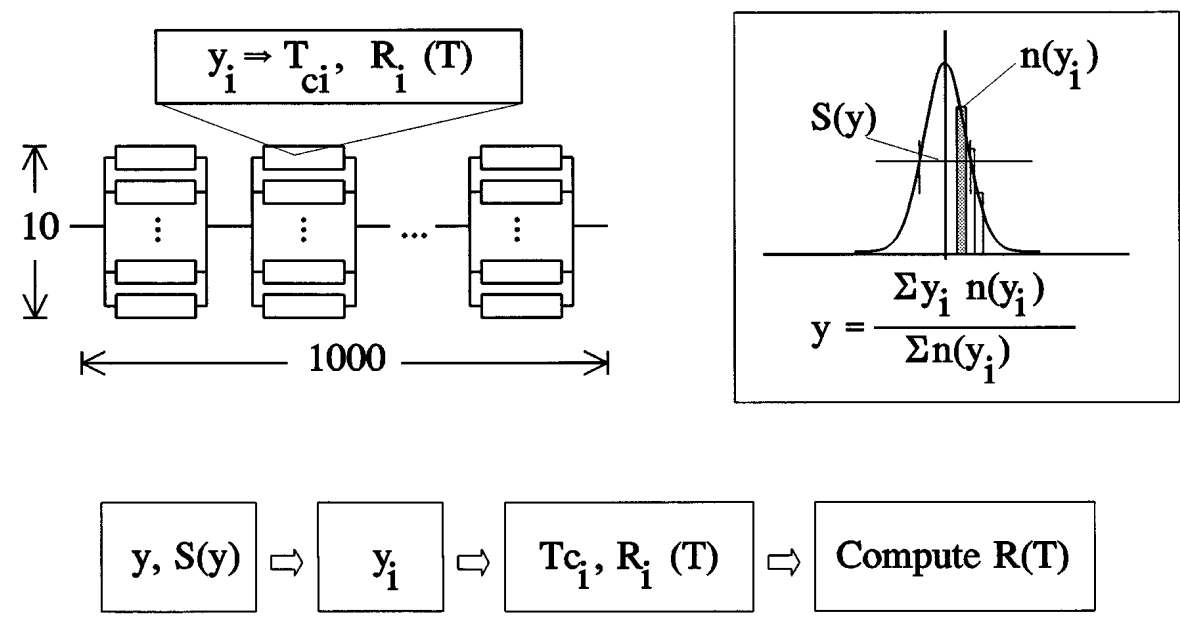

FIG. 4. Diagram of the suggested electrical network for $R(T)$ characteristics simulation. The mean $y$ value and the standard deviation of the $y_{i}$ statistical distribution are the most important input parameters. Two parameters may be added to define $R_{i}(T)$ curves. 


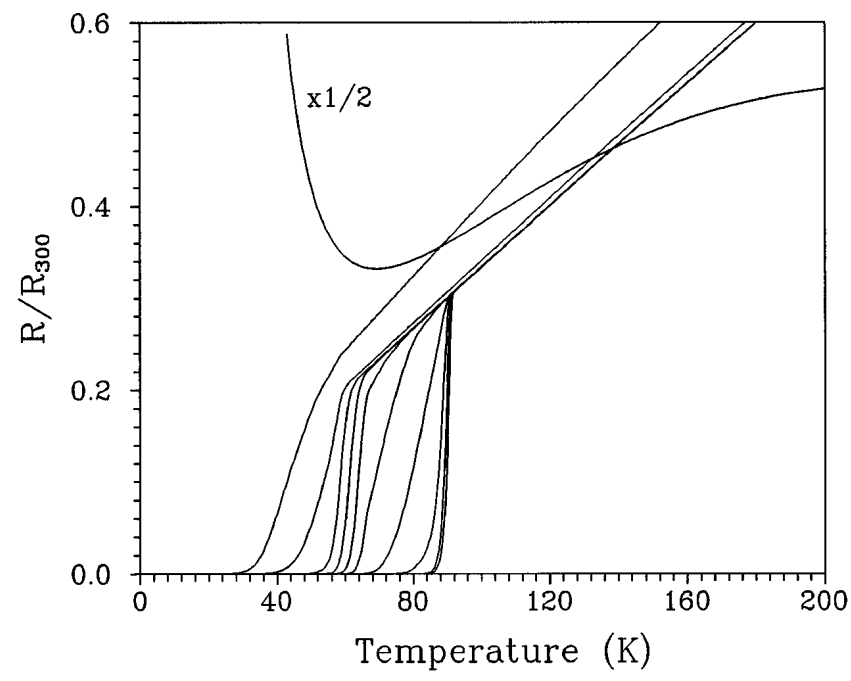

FIG. 5. $R(T)$ curves simulation corresponding to oxygen contents between 6.40 and 6.90 with 0.05 standard deviation. From left to right $y$ increases by 0.05 steps. The $1 / 2$ reduced curve is $y=6.40$.

amount of superconducting material in oxygen-poor or defective areas could be enough to lead to zero resistance in a sample. Unfortunately, the influence of the microstructure on the percolation limit would make it difficult to find out the minimum amount of superconducting material that leads to zero resistance through the whole sample from this model.

When a Gaussian oxygen distribution is used and kept all along the resistor network, as in a long-range uniform sample, the critical temperature rises as the standard deviation of $y$ increases, as far as a minimum superconducting fraction is guaranteed. That occurs because when this deviation rises, more circuit elements are more likely to have higher critical temperatures, leading to an overall resistance decrease at temperatures higher than those expected from the average content.

Granular samples with oxygen-deficient or defective grain boundaries, or other compound precipitates can be studied by combining different phases, conducting or semiconducting. These combinations may affect the normal state behavior, leading to up-rounded curves at high temperatures. Higher activation energies and more semiconducting elements make this effect more pronounced. These cases would account for fine-grained samples or samples with important gaps or microcracks between grains.

This model also shows how the superconducting transition width changes with the oxygen content (Fig. 6). Note that the individual transition width for each element in the network was $0.1 \mathrm{~K}$. The important differences between the curves for different standard deviation values in oxygen distribution, explains the observed scatter in the measured dependence over all the samples (Fig. 3), as far as oxygen distribution is

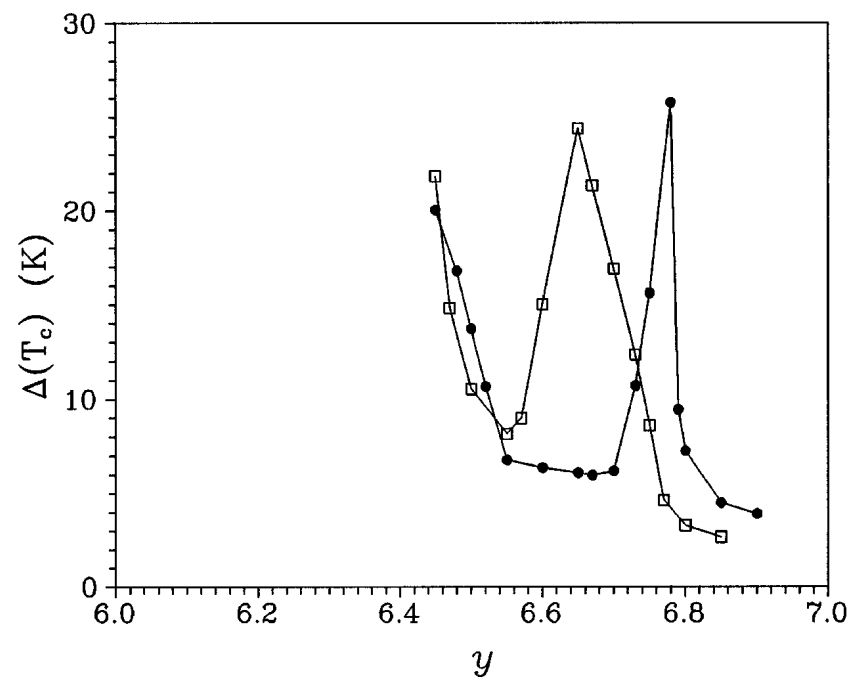

FIG. 6. Simulated dependence of the transition width on the oxygen content. Standard deviation of $y$ is $(\mathbf{O}) 0.05$ and $(\square) 0.10$.

most likely to change with the deposition-annealing process. All these effects are more clearly seen in the derivative curves. The maximum slope point at the superconducting transition rises as the average $y$ increases, and it is usually found at higher temperatures in nonhomogeneous samples. The peak of the $d R(T) / d T$ characteristics is narrow when the oxygen distribution is narrow and almost symmetric when $y$ lies inside a plateau in the $T_{c}-y$ curve. Otherwise, broader and smaller nonsymmetric peaks are found.

The spatial changes in the average oxygen content are specially important. Two samples with equal overall oxygen distributions can lead to quite different $R(T)$ characteristics (Fig. 7), so that the reproducibility of the samples requires a very uniform oxygen incorporation throughout the sample.

\section{Modeling the measured curves}

This model not only reproduces some commonly observed behaviors in $\mathrm{YBa}_{2} \mathrm{Cu}_{3} \mathrm{O}_{y}$ samples, but it also gives a very close approach to the $R(T)$ characteristics in our samples, as can be seen in Fig. 8. The first interesting feature of these results is that the average oxygen values estimated from this model are very close to those measured from XRD data. That means that $y$ values estimated from XRD data, which have been mostly studied for bulk material, can be applied to thin films as far as average oxygen content is concerned. The normal state slope can be reproduced by choosing an appropriate value for residual resistance in the metallic $R(T)$ behavior, giving a measure of amorphous or nonconducting fractions in the sample. The critical temperature and transition widths can also be reproduced 

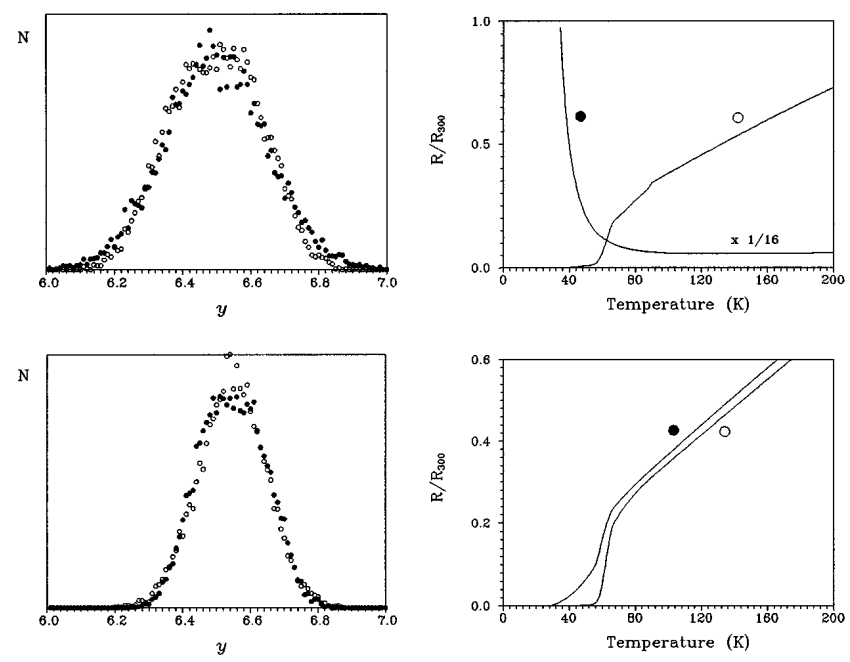

FIG. 7. Effect of oxygen distribution changes along a resistor network. Left: Overall oxygen distribution. Right: $R(T)$ characteristics. The filled dots $(0)$ correspond to variable oxygen distribution along the network, and the open circles $(\bigcirc)$ to constant oxygen distribution all over the network.

by changing $y$ and its standard deviation. In all the cases the elementary transition width is kept as small as possible. In some samples we also reproduce the rounded $R(T)$ characteristics at high temperatures, due to semiconducting areas in the material, with no need to introduce any specific conducting behavior in the high temperature superconducting material.

The rounded shape at temperatures immediately above $T_{c}$ is not reproduced by this model because this shape is mainly due to paraconductivity effects which are not taken into account. But in some cases, rounded curves can be obtained, as it happens in Fig. 8(a) and 8(b). In that case oxygen inhomogeneities would mask or be mixed up with the paraconductivity effects.

\section{CONCLUSION}

Epitaxial $\mathrm{YBa}_{2} \mathrm{Cu}_{3} \mathrm{O}_{y}$ thin films were deposited on $\mathrm{LaAlO}_{3}(100)$ with different oxygen contents through different deposition processes. The samples are epitaxial, and have extremely different electrical properties, depending on the oxygen content $(6 \leqslant y \leqslant 7)$.

The $R(T)$ characteristics change from semiconducting to superconducting with increasing $T_{c}$ values as $y$ rises, following a two-plateau curve. The transition width also changes greatly with the oxygen content. The narrowest transition widths are found in the samples whose $y$ is far away from a phase transition (tetragonal to orthorhombic II and orthorhombic II to orthorhombic I).

A series/parallel resistor model designed to simulate a large mixture of randomly distributed oxygen contents, has been used in order to study the role of oxygen homogeneity in the shape of the $R(T)$ characteristics, (a)
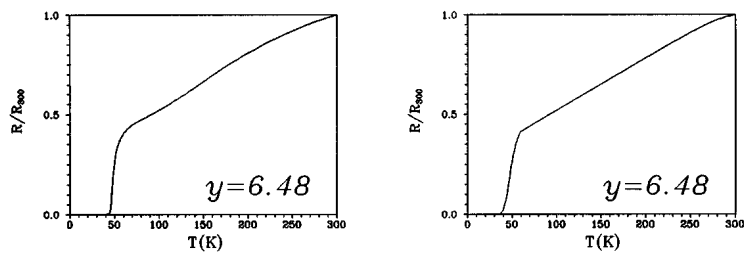

(b)
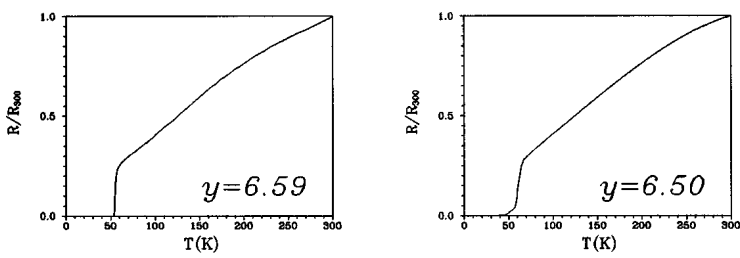

(c)
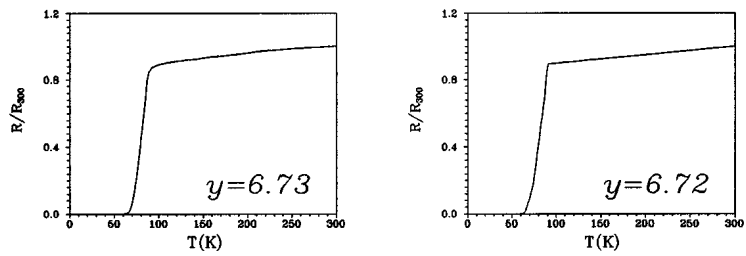

(d)
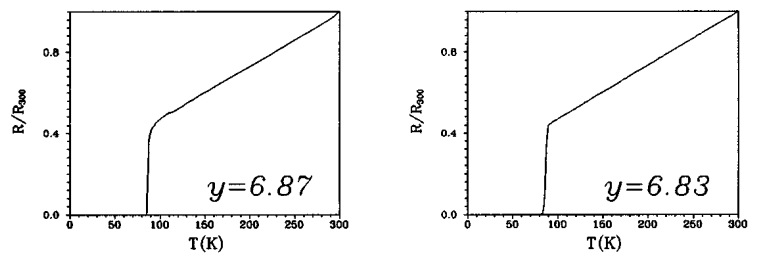

FIG. 8. (a-d) Real (left) and simulated (right) $R(T)$ characteristics of samples with different oxygen contents.

and gives some explanation for measured $R(T)$ curves in $\mathrm{YBa}_{2} \mathrm{Cu}_{3} \mathrm{O}_{y}$ thin films. The model shows the $R(T)$ curves to be dependent not only on the average oxygen content but also on the oxygen distribution, leading to noticeable changes in both transition temperature and width. When the oxygen distributions include semiconducting elements, up-rounded curves at high temperatures can be obtained. Moreover, some distributions can also give a rounded shape just above $T_{c}$, where paraconductivity effects take place, without taking this effect into account. Therefore, an excellent oxygen homogeneity must be provided to study these aspects in the conducting behavior of $\mathrm{YBa}_{2} \mathrm{Cu}_{3} \mathrm{O}_{y}$. The good agreement between the modeled and real $R(T)$ characteristics, with average oxygen contents very close to the values measured from XRD data, could allow us to estimate the oxygen distribution in some samples such as laser-treated and patterned films.

\section{ACKNOWLEDGMENTS}

The authors acknowledge the financial support of CICYT of the Spanish Government (project MAT 93297) and the Generalitat de Catalunya. 


\section{REFERENCES}

1. Y. Iye, in Physical Properties of High Temperature Superconductors III, edited by D. M. Ginsberg (World Scientific Publishing Co. Pte. Ltd., Singapore, 1992), p. 285 and references therein.

2. L. E. Levine and M. Däumling, Phys. Rev. B. 45, 8146 (1992).

3. J. D. Jorgensen, H. Shaked, D. G. Hinks, B. Dabrowski, B.W. Veal, A. P. Paulikas, L. J. Nowicki, G. W. Crabtree, W. K. Kwok, L. H. Nunez, and H. Claus, Physica C 153-155, 578 (1988).

4. E. Takayama-Muromachi, Y. Uchida, M. Ishii, T. Tanaka, and K. Kato, Jpn. J. Appl. Phys. 26, L1156 (1987).

5. R. J. Cava, B. Batlogg, S. A. Sunshine, T. Siegrist, R. M. Fleming, K. Rabe, L. F. Schneemeyer, D. W. Murphy, R. B. van Dover, P. K. Gallagher, S. H. Glarum, S. Nakahara, R. C. Farrow, J. J. Krajewski, S. M. Zahurak, J.V. Waszczak, J.H. Marshall, P. Marsh, L. W. Rupp, W.F. Peck, and E. A. Rietman, Physica C 153-155, 560 (1988).

6. R. Feenstra, D. P. Norton, J. D. Budai, D. K. Christen, D. H. Lowndes, V.C. Mathiasevic, C. B. Eom, T.H. Geballe, E. S. Hellman, and E. H. Hartford, in Layered Superconductors: Fabrication, Properties and Applications, edited by D. T. Shaw, C. C. Tsuei, T. R. Schneider, and Y. Shiohara (Mater. Res. Soc. Symp. Proc. 275, Pittsburgh, PA, 1992), p. 101.

7. E. C. Jones, D. K. Christen, J. R. Thompson, R. Feenstra, S. Zhu, D. H. Lowndes, J. M. Phillips, and J.P. Siegal, Phys. Rev. B 47, 8986 (1993).
8. C. Thomsen, R. Wegerer, H. U. Habermeier, and M. Cardona, Solid State Commun. 83, 199 (1992).

9. A. T. Fiory, S. Martin, L. F. Schneemeyer, R. M. Fleming, A.E. White, and J. V. Waszczak, Phys. Rev. B 38, 7129 (1988).

10. R. J. Cava, A. W. Hewat, E. A. Hewat, B. Batlogg, M. Marezio, K. M. Rabe, J. J. Krajewski, W.F. Peck, and L. W. Rupp, Physica C 165, 419 (1990).

11. J. M. Tranquada, S. M. Heald, A. R. Moodenbaugh, and Y. Xu, Phys. Rev. B 38, 8893 (1988).

12. C. Champeaux, P. Marchet, J. Aubreton, J.P. Mercurio, and A. Catherinot, Appl. Surf. Sci. 69, 335 (1988).

13. J. Ye and K. Nakamura, Phys. Rev. B 48, 7554 (1993).

14. K. Maki, Progr. Theor. Phys. 39, 897 (1968).

15. R. S. Thompson, Phys. Rev. B 1, 327 (1970).

16. L. G. Aslamazov and A. I. Larkin, Phys. Lett. A 26, 238 (1968).

17. G. F. Dionne, IEEE Trans. Magn. 27 (2), 1190 (1991).

18. G. F. Dionne, J. Appl. Phys. 69 (8), 4883 (1991).

19. A. Kreisler, F. Hosseini Teherani, J. M. Depond, and J. Baixeras, in High $T_{c}$ Superconductor Thin Films, edited by L. Correra (Elsevier Science Publishers, Amsterdam, 1992), p. 131.

20. R. J. Thorn, Physica C 190, 193 (1992).

21. K. Yamamoto, B. M. Lairson, J.C. Bravman, and T.H. Geballe, J. Appl. Phys. 69, 7189 (1991).

22. R. J. Cava, B. Batlogg, K. M. Rabe, E. A. Rietman, P. K. Gallagher, and L. W. Rupp, Physica C 156, 523 (1988). 